\title{
Study on Biased Multi Arch Tunnel Biasing Angle Effect on Mechanical Behavior of Surrounding Rock Bias
}

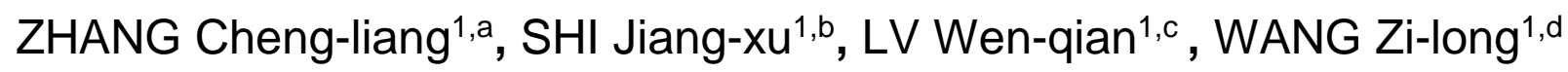

${ }^{1}$ Faculty of Land Resources Engineering, Kunming University of Science and Technology, Kunming, Yunnan, China 650093

azclky78@163.com, b1330983842@qq.com, '864788235@qq.com, d1543207383@qq.com

\section{Keywords: Multi arch tunnel, Terrain bias, Bias angle, Bias characteristic.}

Abstract. The exits and entrances of the multi arch tunnel are often located in unfavorable geological sections, such as shallow buried, bias, high weathering degree, rock breaking and so on. In addition, the existence of the side slope makes the force more complicated after the tunnel excavation. When exits and entrances of tunnels are excavated, there are often large deformation of surrounding rock, initial deformation and two lining cracks. Serious accidents may lead to collapse and instability. Based on the geological conditions and the mechanical characteristics of the surrounding rock, the influence of the bias angle of the biased multi arch tunnel on the mechanical properties of the surrounding rock after excavation is studied by the theoretical analysis and numerical simulation. It is said that with the increase of the bias angle; the influence of the deep buried side of the bias tunnel is more obvious than that of the shallow buried side. Numerical simulation of excavation of a multi arch tunnel under different bias angles is carried out. According to the analysis of the critical point displacement, the influence of the tunnel under the bias pressure intensified as the slope go steeper. And when the thickness of the earth beside is over 15 meters, the influence of it would get rids of the change of different bias angle. These results can provide some reference and guidance for similar arch tunnel design, construction and research.

\section{Introduction}

Owing to the promotion and implementation of "Weastern Development" and "Belt and Road Initiative" development strategy [1-2], the western mountainous areas came to be the core area of traffic construction process. In the past, most of the roads built in the western mountainous areas were mostly built as winding roads because of the bad traffic construction conditions. The highway was unable to meet the requirements of the contemporary highway projects because of its shortages such as low grade, long mileage, occupying more arable land, poor driving safety performance and doing harm to the ecological environment [3-4]. Therefore, highway tunnels are widely used in highway construction such as improving highway linearity, shortening mileage, protecting ecological environment and saving cultivated land thanks to their advantages. However, the entrance of the multi arch tunnel are often buried in the bad geological section, such as shallow burial, bias, high weathering, rock breaking and so on. This causes the tunnel exit and entrance to be in a complex stress area[5], and the existence of the side slope lead to the more complicated force environment after the tunnel excavation. Excavation of tunnels often results in accidents such as excessive deformation of surrounding rocks, collapse, and instability. Cai Zhiwei et al[6]. used the numerical simulation of Tongji dawning finite element software for a large section continuous arch tunnel, and analyzed the stress and strain changes of the surrounding rock and the supporting structure when the tunnel was excavated by the three guide tunnel method. Shen Yusheng et al[7]. monitored and analyzed the double arch tunnel through field monitoring, and analyzed the bias characteristics of the tunnel support structure under construction bias condition. Liu Xiaojun et al[8]. carried out numerical simulation research on shallow buried partial pressure tunnel through FLAC3D software, and compared this two different construction processes: starting with shallow side excavation or starting with deep side excavation, analysed and found the best process. In the paper, taking the shallow buried section of the Qi Fengshan bias arch tunnel as the research object, the mechanical properties of 
the surrounding rock under different bias pressure are studied. The results can provide some reference and guidance for similar arch tunnel design, construction and research.

\section{Engineering situation}

The left and right range of the Qi Fengshan tunnel in the central section of the Shuang yue road is $\mathrm{K} 4+915$ to $\mathrm{K} 5+290$, and the length is $375 \mathrm{~m}$; the maximum depth of the tunnel is about $48 \mathrm{~m}$, and the longitudinal slope of the tunnel is $-0.617 \%$. The right amplitude exit of the Qi Fengshan tunnel is located in the shallow buried section. The weathering of the overlying layer of the tunnel is serious, the rock and soil mass are broken and the surrounding rock integrity is poor. The design of surrounding rock in the tunnel area is mainly based on the V level. The tunnel is a two-way three car multi arch tunnel with a span of $15.23 \mathrm{~m}$ and a height of $7.65 \mathrm{~m}$. In the statistics of the central area tunnel, the bias angle is 16 places from 21 to 50 degrees, accounting for $80 \%$ of the total, and the slope of the slope from 20 to 40 degrees is 16 places, accounting for $80 \%$ of the total. In all the entrance and exit sections of the tunnel, there are 14 places in the shallow buried cases, which account for $70 \%$ of the total. Most of the shallow buried tunnels have shallow bias or shallow buried severe bias. Statistics of topography and geological conditions of Qi Fengshan tunnel are shown as Table 1.

Table 1 Topographic geological statistics

\begin{tabular}{|c|c|c|c|c|c|c|}
\hline $\begin{array}{c}\text { Tunnel } \\
\text { name }\end{array}$ & & & $\begin{array}{c}\text { Grade of } \\
\text { surrounding } \\
\text { rock }\end{array}$ & Bias angle & $\begin{array}{l}\text { Slope } \\
\text { slope }\end{array}$ & Buried depth \\
\hline \multirow{4}{*}{$\begin{array}{c}\text { Qi } \\
\text { Fengshan } \\
\text { tunnel }\end{array}$} & \multirow{2}{*}{$\begin{array}{c}\text { Left } \\
\text { amplitude }\end{array}$} & Imported & $\mathrm{V}$ & $45^{\circ}$ & $19^{\circ}$ & $(0 \sim 48) \mathrm{m}$ \\
\hline & & Exit & V & $45^{\circ}$ & $19^{\circ}$ & $(0 \sim 48) \mathrm{m}$ \\
\hline & \multirow{2}{*}{$\begin{array}{c}\text { Right } \\
\text { amplitude }\end{array}$} & Imported & $\mathrm{V}$ & $35^{\circ}$ & $24^{\circ}$ & $(0 \sim 48) \mathrm{m}$ \\
\hline & & Exit & $\mathrm{V}$ & $35^{\circ}$ & $24^{\circ}$ & $(0 \sim 48) \mathrm{m}$ \\
\hline
\end{tabular}

\section{The calculation of the bias load}

The topographic bias of the tunnel section of the shallow buried multi-arch tunnel is relatively obvious, and the tunnel lining load can be calculated as follows[9].It is assumed that the distribution pattern of tunnel bias is consistent with that of the ground slope.

$$
Q=\frac{\gamma}{2}\left[\left(h+h^{\prime}\right) B-\left(\lambda h^{2}+\lambda^{\prime} h^{\prime 2}\right) \tan \theta\right] .
$$

h、h - The height of the inner and outer sides from the vault to the ground; B - Tunnel width; $\theta$ Friction angles on both sides of the roof soil column $\left(^{\circ}\right) ; \gamma$ - The severity of the surrounding $\operatorname{rock}\left(\mathrm{KN} / \mathrm{m}^{2}\right)$.

Table 2 Angle of friction angle

\begin{tabular}{clccc}
\hline Grade of surrounding rock & I $\sim$ III & IV & V & VI \\
\hline$\theta$ & $0.9 \varphi_{c}$ & $(0.7 \sim 0.9) \varphi_{c}$ & $(0.5 \sim 0.7) \varphi_{c}$ & $(0.3 \sim 0.5) \varphi_{c}$ \\
\hline
\end{tabular}

$\lambda 、 \lambda$ '-The side pressure coefficient of the inner and outer sides is calculated by the lower formula:

$$
\begin{gathered}
\lambda=\frac{1}{\tan \beta-\tan \alpha} \times \frac{\tan \beta-\tan \varphi_{c}}{1+\tan \beta\left(\tan \varphi_{c}-\tan \theta\right)+\tan \varphi_{c} \tan \theta} . \\
\lambda^{\prime}=\frac{1}{\tan \beta^{\prime}+\tan \alpha} \times \frac{\tan \beta^{\prime}-\tan \varphi_{c}}{1+\tan \beta^{\prime}\left(\tan \varphi_{c}-\tan \theta\right)+\tan \varphi_{c} \tan \theta} .
\end{gathered}
$$




$$
\begin{aligned}
& \tan \beta=\tan \varphi_{c}+\sqrt{\frac{\left(\tan ^{2} \varphi_{c}+1\right)\left(\tan \varphi_{c}-\tan \alpha\right)}{\tan \varphi_{c}-\tan \theta}} . \\
& \tan \beta^{\prime}=\tan \varphi_{c}+\sqrt{\frac{\left(\tan ^{2} \varphi_{c}+1\right)\left(\tan \varphi_{c}+\tan \alpha\right)}{\tan \varphi_{c}-\tan \theta}} .
\end{aligned}
$$

$\alpha$ - Slope angle of ground $\left(^{\circ}\right) ; \varphi_{c}$ - Calculation of friction angle of surrounding $\operatorname{rock}\left(^{\circ}\right) ; \beta$ 、 $\beta^{\prime}$ - Break angle when the inner and outer sides produce the maximum thrust $\left(^{\circ}\right)$.

Lateral pressure:

$$
e_{i}=\gamma \cdot h_{i} \lambda
$$

Calculation of vertical pressure under loading:

$$
q_{0}=\frac{Q}{B}
$$

\section{Analysis of relationship between pressure and bias angle of bias tunnel}

According to the formula of the eccentric load calculation, the height of the tunnel vault to the surface is kept constant, and the discipline of the surrounding rock and bias angle in tunneling is studied.

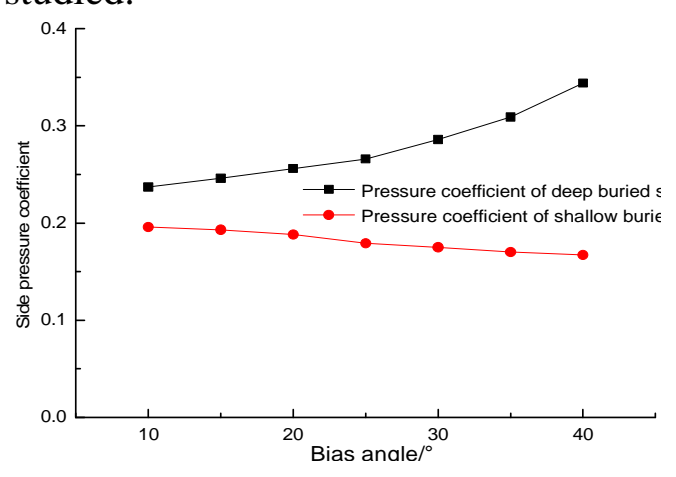

\begin{tabular}{ccc}
\hline \multirow{2}{*}{ Bias angle $\left({ }^{\circ}\right)$} & \multicolumn{2}{c}{ Side pressure coefficient } \\
\cline { 2 - 3 } & $\lambda$ & $\lambda^{\prime}$ \\
\hline 10 & 0.237 & 0.196 \\
15 & 0.246 & 0.193 \\
20 & 0.256 & 0.188 \\
25 & 0.266 & 0.179 \\
30 & 0.286 & 0.175 \\
35 & 0.309 & 0.17 \\
40 & 0.344 & 0.167 \\
\hline
\end{tabular}

(a)The relationship between the bias angle and the side pressure coefficient

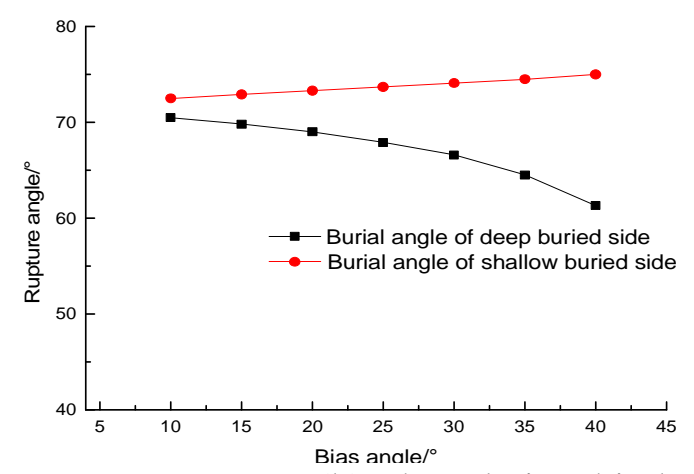

\begin{tabular}{ccc}
\hline \multirow{2}{*}{ Bias angle $\left({ }^{\circ}\right)$} & \multicolumn{2}{c}{ Rupture angle $\left(^{\circ}\right)$} \\
\cline { 2 - 3 } & $\beta$ & $\beta$ \\
\hline 10 & 70.5 & 72.5 \\
15 & 69.8 & 72.9 \\
20 & 69.0 & 73.3 \\
25 & 67.9 & 73.7 \\
30 & 66.6 & 74.1 \\
35 & 64.5 & 74.5 \\
40 & 61.3 & 74.99 \\
\hline
\end{tabular}

(b) The relationship between the bias angle and the rupture angle 


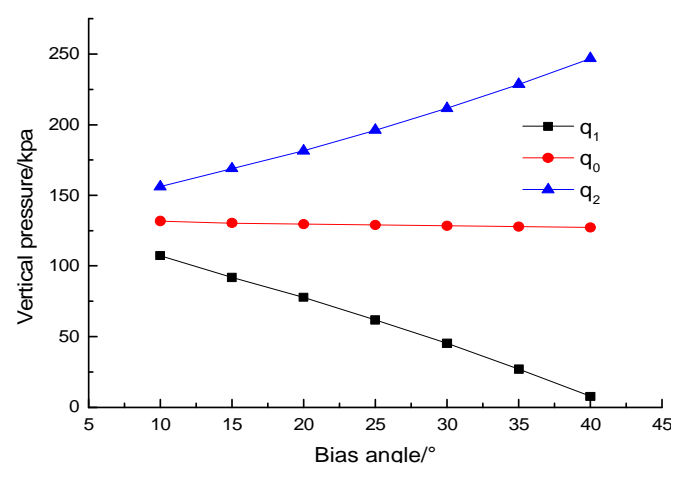

\begin{tabular}{cccc}
\hline Bias & \multicolumn{3}{c}{ Vertical pressure(kpa) } \\
\cline { 2 - 4 } angle $\left({ }^{\circ}\right)$ & $\mathrm{q}_{1}$ & $\mathrm{q}_{0}$ & $\mathrm{q}_{2}$ \\
\hline 10 & 107.3 & 131.8 & 156.2 \\
15 & 91.8 & 130.4 & 168.9 \\
20 & 77.8 & 129.7 & 181.5 \\
25 & 61.8 & 129 & 196.1 \\
30 & 45.3 & 128.5 & 211.6 \\
35 & 27 & 127.8 & 228.6 \\
40 & 7.7 & 127.3 & 246.8 \\
\hline
\end{tabular}

(c)The relationship between the bias angle and the vertical pressure

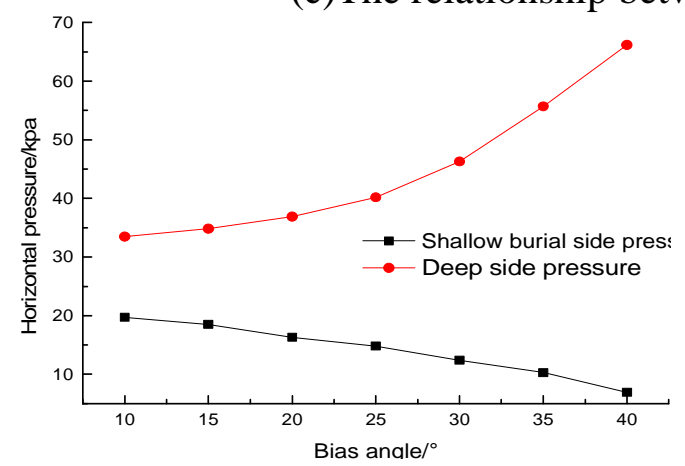

\begin{tabular}{ccc}
\hline \multirow{2}{*}{ Bias angle $\left({ }^{\circ}\right)$} & \multicolumn{2}{c}{ Horizontal pressure $(\mathrm{kpa})$} \\
\cline { 2 - 3 } $\mathrm{e}_{1}$ \\
\hline 10 & 19.7 & 33.5 \\
15 & 18.5 & 34.8 \\
20 & 16.3 & 36.9 \\
25 & 14.8 & 40.2 \\
30 & 12.4 & 46.3 \\
35 & 10.3 & 55.7 \\
40 & 6.9 & 66.2 \\
\hline
\end{tabular}

(d) The relationship between the bias angle and the horizontal pressure

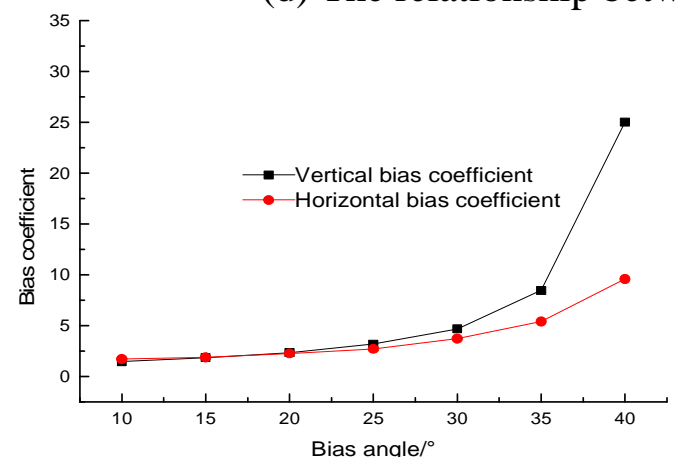

\begin{tabular}{ccc}
\hline \multirow{2}{*}{ Bias angle $\left(^{\circ}\right)$} & \multicolumn{2}{c}{ Bias coefficient } \\
\cline { 2 - 3 } & $\mathrm{K}_{\mathrm{v}}$ & $\mathrm{K}_{\mathrm{h}}$ \\
\hline 10 & 19.7 & 33.5 \\
15 & 18.5 & 34.8 \\
20 & 16.3 & 36.9 \\
25 & 14.8 & 40.2 \\
30 & 12.4 & 46.3 \\
35 & 10.3 & 55.7 \\
40 & 6.9 & 66.2 \\
\hline
\end{tabular}

(e) The relationship between bias angle and bias coefficient

Fig. 1 The relationship between the bias angle and the parameters of the surrounding rock pressure

From the analysis of Fig. 1, it can be seen that when the height of the tunnel vault to the surface is kept constant. (1) When the angle of the tunnel bias increases, the pressure coefficient of the deep buried side increases gradually, and the pressure coefficient of the shallow buried side decreases gradually. This shows that with the increase of the bias angle, the bias pressure has a significant effect on the deep buried side of the tunnel, and the influence of the bias on the shallow buried side of the tunnel is impaired. (2) With the increase of the bias angle, the rupture angle of the deep buried side increases and the rupture angle of the shallow buried side decreases. This shows that under the increase of the bias angle, the range of the bias affected by the deep buried side increases, and the shallow buried side decreases. It can be seen from the calculation formula that the angle of the rupture angle is only related to the bias angle, but has nothing to do with the tunnel depth. (3) In the vertical direction, the total stress of the vault decreases slightly with the increase of the bias angle, the vertical stress of the deep buried side increases rapidly, and the vertical stress of the shallow buried side decreases rapidly. In horizontal direction, with the increase of the bias angle, the horizontal stress of deep buried side also increases, while as the horizontal stress of shallow buried side decreases. (4) Under the condition of constant total stress of the vault, with the increase of the bias angle, the stress shared by the deep buried side is increasing, and the stress shared by the shallow side is decreasing. 


\section{Numerical simulation analyses of bias characteristics under different bias angles}

Under the condition of $\mathrm{V}$ grade, the influence of bias pressure on the mechanical properties of surrounding rock under eccentric loading angle of 25, 35 and 45 degrees is studied. The displacement data with different soil thickness are simulated under different working conditions, and the displacement data of different bias angles are obtained. Take the displacement of the vault, the horizontal displacement of the right spandrel and the maximum outward displacement of the slope table as the key points to analyze and draw the curves as shown in Fig. 4.
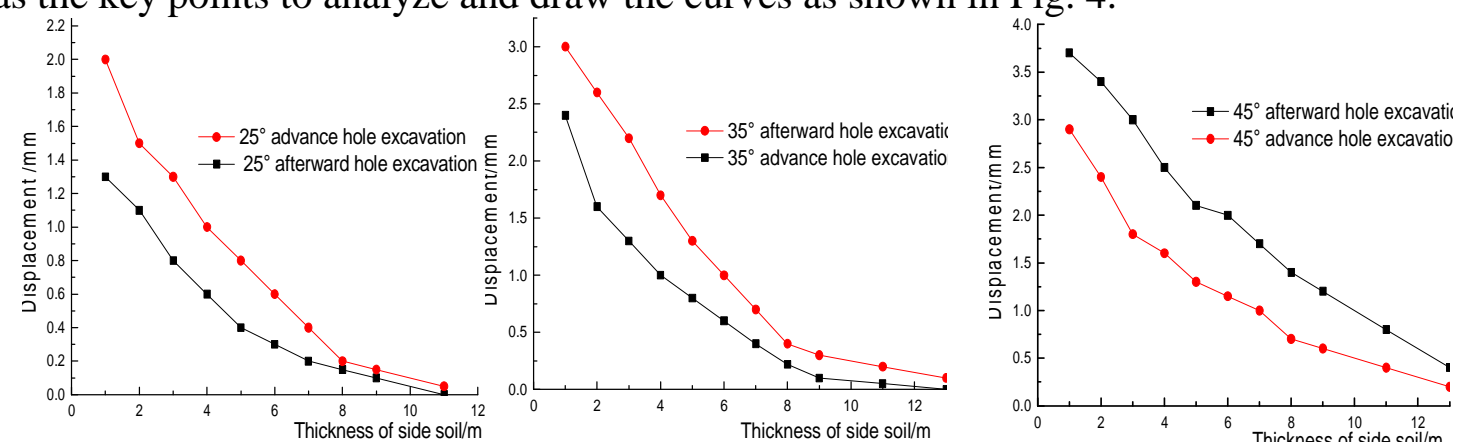

(a)The maximum displacement diagram of the slope surface

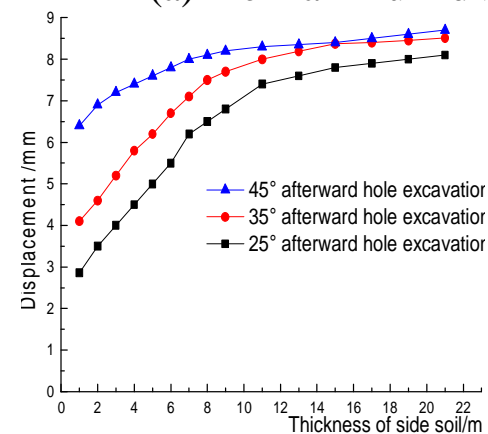

(b) Vertical displacement

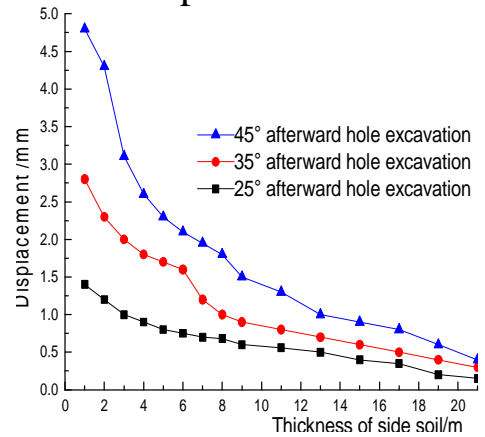

(c) Horizontal displacement of the right arch shoulder

Fig. 2 Displacement cloud chart and displacement diagram of multi arch tunnel with different bias angles

From the analysis of Fig. 2, (1) When the side thickness of soil is same, the larger the bias angle is, the greater the vertical displacement is. When the angle of bias is 25 degrees, the speed of increasing with the thickness of the side overlying soil is the fastest, and the angle of bias pressure is 35 and 45 degrees decreasing in turn. This is because the increase of the thickness of the overlying soil will also increase the thickness of the upper soil layer, which has a great influence on the vertical displacement of the cave body. When the angle of the partial pressure is large, the thickness of the upper layer of the tunnel is very large. At the same time, the increase of lateral soil thickness has little effect on the thickness of the soil layer. And the effect on the vertical displacement of the tunnel is relatively small. (2) When the side thickness of soil is same, the greater the bias angle, the greater the maximum outward displacement of the slope surface and the horizontal displacement of the right spandrel. (3) Under the different bias angle, When the thickness of the overlying soil on the side of the multi-arch tunnel exceeds $15 \mathrm{~m}$, the vertical displacement, horizontal displacement, and maximum outward displacement of the slope surface on the surface of the shallow-buried cavern have small changes and gradually tend to be stable.

\section{Conclusion}

By theoretically and numerically analyzing the bias characteristics of a biased double-arch tunnel, the research results are as follows.

(1)When the tunnel bias angle is increased, the influence of the bias on the deep-buried side of the tunnel is significantly enhanced; the fracture angle on the deep-buried side is increased, and from the formula for the fracture angle, it can be seen that the angle of the fracture angle is only related to the bias angle, but not related to the tunnel depth. 
(2)With the increase of the bias angle, the total stress of the vault decreases slightly; the vertical stress of the deep buried side increases rapidly; the lateral stress of the deep buried side also increases. Under the constant total stress of the vault, the stress shared by the deep buried side is increasing.

(3)Through the analysis of the displacement of key points, it is found that under the same thickness of the overlying soil, the greater the deflection angle, the greater the vertical displacement, the horizontal displacement and the maximum displacement of the slope surface. Under different biasing angles, when the thickness of the side covering soil exceeds $15 \mathrm{~m}$, the shallow-buried soil can already provide effective back pressure. At this time, the influence of the bias on the whole body can be considered to be very small. When the thickness of the side covering soil is less than $15 \mathrm{~m}$, the whole body is greatly affected by the bias angle. The safety treatment measures should be considered.

\section{Acknowledgements}

This work was financially supported by the National Natural Science Foundation of China (51264018).

\section{References}

[1] Ma Xin Wen. The western development of the West should be guided by the transportation industry as the leader of the [A]. Shaanxi Province Economics Society. The Twentieth Annual Conference and Theoretical Symposium of the Shaanxi Provincial Economic Society, [C]. Economic Society of Shaanxi Province: 2000:7.

[2] Dong Suocheng, Cheng Hao, Guo Peng, Li Fujia, Li Yu, Li Zehong, Zhang Xiaoxiao. "The industry pattern and Countermeasures of transportation The Belt and Road",[J]. Journal of the Academy of Chinese Academy of Sciences, 2016, (06): 663-670.

[3] Hu Shi. Analysis of the current situation and matters needing attention in mountainous highway design [J]. China Management Informatization, 2015, (05): 172-173.

[4] Hou Ling. Safety evaluation of key sections of expressways in western mountainous areas [D]. Chang'an University, 2014.

[5] Zhang Jinpeng. Stability analysis and prevention engineering study of landslide at the entrance of Yang Po Li tunnel [D]. LanZhou JiaoTong University, 2012.

[6] Cai Zhiwei, Lin Zhi. Analysis of the force and deformation law of surrounding rock and middle wall of large section multi arch tunnel [C]// Annual Meeting of China Civil Engineering Society. 2006.

[7] Shen Yusheng. Monitoring and analysis of mechanical properties of high pressure double arch tunnel under construction bias [J]. Rock and Soil Mechanicss, 2006, 27 (11): 2061-2065.

[8] Liu Xiaojun, Zhang Yongxing. Reasonable excavation procedure and stress characteristics of shallow buried bias tunnel portal section [J]. Chinese Journal of Rock Mechanics and Engineering, 2011 (S1): 3066-3073.

[9] Code for design of railway tunnels (TB10003-2001). China Railway Publishing House.2001. 